\title{
LA-UR- $08-5928$
}

Approved for public release;

distribution is unlimited.

On the Extent and Connectivity of Conical Intersection Seams and the Effects of Three-State Intersections

Author(s):

Joshua D. Coe, Mitchell T. Ong, Benjamin G. Levine, and Todd J. Martinez

Intended for:

Journal of Physical Chemistry A

EST.1943

Los Alamos National Laboratory, an affirmative action/equal opportunity employer, is operated by the Los Alamos National Security, LLC for the National Nuclear Security Administration of the U.S. Department of Energy under contract DE-AC52-06NA25396. By acceptance of this article, the publisher recognizes that the U.S. Government retains a nonexclusive, royalty-free license to publish or reproduce the published form of this contribution, or to allow others to do so, for U.S. Government purposes. Los Alamos National Laboratory requests that the publisher identify this article as work performed under the auspices of the U.S. Department of Energy. Los Alamos National Laboratory strongly supports academic freedom and a researcher's right to publish; as an institution, however, the Laboratory does not endorse the viewpoint of a publication or guarantee its technical correctness.

Form $836(7 / 06)$ 


\title{
On the Extent and Connectivity of Conical Intersection Seams and the Effects of Three-State Intersections
}

\author{
Joshua D. Coe, Mitchell T. Ong, Benjamin G. Levine, and Todd J. Martínez \\ Department of Chemistry, Beckman Institute, and Frederick Seitz \\ Materials Research Laboratory, University of Illinois at Urbana- \\ Champaign, 600 S. Mathews Ave., Urbana, IL 61801.
}

\begin{abstract}
We discuss the connectivity of intersection spaces and the role of minimal energy points within these intersection spaces (minimal energy conical intersections or MECIs) in promoting nonadiabatic transitions. We focus on malonaldeyde as a specific example, where there is a low-lying three-state conical intersection. This three-state intersection is the global minimum on the bright excited electronic state, but it plays a limited role in population transfer in our ab initio multiple spawning (AIMS) simulations because the molecule must traverse a series of two-state conical intersections to reach the three-state intersection. Due to the differences in seam space dimensionality separating conventional (two-state) and three-state intersections, we suggest that dynamical effects arising directly from a three-state intersection may prove difficult to observe in general. We also use a newly developed method for intersection optimization with geometric constraints to demonstrate the connectivity of all the stationary points in the intersection spaces for malonaldehyde. This supports the conjecture that all intersection spaces are connected, and that three-state intersections play a key role in extending this connectivity to all pairs of states, e.g. the $\mathrm{S}_{1} / \mathrm{S}_{0}$ and $\mathrm{S}_{2} / \mathrm{S}_{1}$ intersection spaces.
\end{abstract}

Coe, Levine, Ong, and Martinez - Page 1 


\section{Introduction}

Light-mediated chemistry offers the prospect of revolutionary and diverse applications such as coherent control, ${ }^{1,2}$ rationally designed molecular scale devices, ${ }^{3-5}$ and the efficient hamessing of solar energy. ${ }^{6}$ Progress in any of these endeavors requires understanding the evolution and decay of electronically excited states. Almost from the advent of quantum mechanics, it was known that Born-Oppenheimer surfaces become nearly degenerate at select nuclear configurations. ${ }^{8}$ Such points, dubbed avoided crossings, rightfully were identified closely with electronic relaxation. Soon after, it was realized that for polyatomic molecules there may also exist geometries where the energy gap vanishes completely. These conical intersections, ${ }^{10,11}$ nuclear configurations for which a pair of electronic surfaces becomes perfectly degenerate, have come to play a pivotal role in most discussions of electronically excited states. ${ }^{3,12-14}$

It is well known that conical intersections are not isolated points, but rather highdimensional "seams." Visualization of these high-dimensional objects is very difficult. Thus they often have been characterized by one or more representative lowest-energy points within the "intersection space," molecular configurations known as minimal energy conical intersections (MECIs). The relevance of such geometries to electronic excited state dynamics has been a recurring question - can these MECIs be considered as the analogues of transition states, i.e. as bottlenecks in the electronic quenching leading from an upper electronic state to a lower electronic state? Or is it instead necessary to consider the geometry of the entire intersection space? An additional wrinkle has been introduced by the recent realization that not only two-state conical intersections, but also three-state conical intersections (geometries where three electronic states become 
degenerate) are often accessible at energies below the Franck-Condon point. ${ }^{15-20}$ How does the presence of three-state intersections affect electronic quenching dynamics and the topology of the intersection space?

Perhaps the first question to answer regarding intersection space topology relates to connectivity. We have conjectured previously ${ }^{21}$ that all MECIs connecting a pair of electronic states are reachable by all other MECls (connecting the same pair of electronic states) through a path lying entirely within the intersection space. In other words, that the intersection space for a given pair of states is fully connected. We have further hypothesized that three-state intersections play a key role in extending this "connectivity conjecture" to intersection spaces joining any pair of electronic states. Under these circumstances, the entire intersection space is fully connected, e.g., points lying on the $S_{0} / S_{1}$ seam are reachable from points lying on the $S_{2} / S_{1}$ seam via paths that link the two intersection spaces through three-state $\mathrm{S}_{2} / \mathrm{S}_{1} / \mathrm{S}_{0}$ conical intersections. Ultimately, a rigorous proof (or disproof) of this conjecture is desirable. However, until such a proof is available, explorations of intersection space connectivity provide evidence for the veracity of the conjecture.

In this paper, we use malonaldehyde as a test case in which to probe these issues. Malonaldehyde is the paradigmatic molecule for excited state intramolecular proton transfer (ESIPT), an important and well-studied photochemical process in its own right. ${ }^{22-}$ ${ }^{30}$ However, our present interest centers more on ${ }^{19,20,31}$ l) there existing two chemically distinct conical intersections thought to be relevant to decay of the $S_{2}$ bright state in this molecule, 2) one of these intersections being a low-lying three-state $\mathrm{S}_{2} / \mathrm{S}_{1} / \mathrm{S}_{0}$ intersection 
that is also the lowest energy point on $S_{2}$ and 3) the molecule being small enough to permit extensive sampling.

The content of the present work is threefold. First, we use $a b$ initio multiple spawning (AIMS) dynamics to examine the correlation of population flow with distance from the seam minimum. The potential dynamical relevance of a seam's extent, in addition to its point of minimal energy, has been noted previously, ${ }^{10,32-34}$ and intersection of the seam space with the steepest descent pathway on the upper electronic surface can promote relaxation "localized" at segments of the seam other than its minimum. ${ }^{35}$ The present work enlarges upon this theme, and shows, surprisingly, that neither of the abovementioned conical intersection geometries (which are stationary points within the seam space) provides a good representation of geometries leading to nonadiabatic transitions. This contrasts sharply with the common portrayal of MECls as the dominant locus of quenching along a seam.

Secondly, we link two conical intersection extrema of very different chemical character without breaking the degeneracy at any point along the connecting pathway using a recently developed intersection optimization method incorporating geometric constraints. This allows us to show that only one of these points is truly an $\mathrm{MECl}$ - the other is an intersection space saddle point whose precise order has yet to be determined. $33,36,37$

Finally, we examine the extent to which 3-state intersections participate directly in electronic relaxation. After clarifying the relative significance of the minimal energy three state intersection (ME-3SI) for $\mathrm{S}_{2} \rightarrow \mathrm{S}_{1}$ internal conversion in malonaldehyde, we examine its role in ground state recovery. Although three-state intersections in methyl

Coe, Levine, Ong, and Martinez-Page 4 
cation $^{38}$ were examined much earlier, recent discussions canvas their occurrence in ethyl, ${ }^{16}$ allyl, ${ }^{17,39}$ and pyrazolyl radicals, ${ }^{18,40-44}$ butadiene, ${ }^{45}$ and in the nucleic acid bases cytosine $^{46}$ uracil, $^{15}$ and adenine. ${ }^{15}$ General analysis of the seam space, ${ }^{41,42}$ geometric phase, ${ }^{39,47}$ and algorithms for 3 -state seam minimization ${ }^{16,45}$ have also been reported. Apart from a single preliminary study, ${ }^{19}$ however, exploration of the role played by threestate intersections in the context of molecular dynamics has been lacking.

What follows is a series of observations regarding conical intersections and their role in nonadiabatic dynamics of polyatomic molecules. Although points are adduced from evidence in malonaldehyde, we believe that underlying each are principles whose validity can be extended to many other systems with only minor modification.

\section{Methods}

Intersection connectivity is demonstrated using an optimization scheme detailed previously, ${ }^{45,48}$ but adapted to include geometric constraints. The algorithm utilizes a Lagrange multiplier penalty method, ${ }^{49}$ and is able to locate minima along an intersection seam without knowledge of the nonadiabatic coupling vector. Derivative discontinuities are smoothed with a penalty function $G$ which remains smooth even in the neighborhood of a discontinuity in the gradient of the potential surface. The objective function to be minimized is:

$$
L_{L J}(\mathbf{R} ; \sigma, \lambda, \alpha)=\bar{E}_{I J}(\mathbf{R})+\frac{1}{2} \sigma G\left(\Delta E_{I J}(\mathbf{R}) ; \alpha\right)+\sum_{n=1}^{N_{g e v}} \lambda^{(n)}\left(\phi^{(n)}(\mathbf{R})-\phi_{0}^{(n)}\right)
$$

where $I$ and $J$ label electronic states with average energy $\bar{E}_{I J}$ and energy difference $\Delta E_{I J}$,

$\mathbf{R}$ denotes the full range of molecular coordinates, and $\phi^{(n)}(\mathbf{R})$ denotes one of the $N_{\text {geo }}$ 
internal coordinates which is to be constrained to the value $\phi_{0}^{(n)}$. The Lagrange multipliers $\lambda^{(n)}$ enforce the geometric constraints, the smoothing parameter $\alpha$ ensures well-behaved surfaces even near conical intersections, and $\sigma$ is a sequentially updated parameter driving the optimization toward the seam space minimum. In the work discussed here, we constrain a single dihedral angle $\left(\phi_{i j k i}\right)$ spanning atoms $i, j, k$, and $l$ to the value $\phi_{0}$, but adaptation to bond or angle constraints is obvious. Use of the smoothing function $G$ is similar in spirit to Yarkony's use of extrapolatable functions, ${ }^{50,51}$ but his are within the context of a Newton-Raphson scheme requiring nonadiabatic couplings and Hessian updating. The specific form of $G$ used in the present work is

$$
G=\frac{\Delta E_{W}^{2}}{\Delta E_{\mu}+\alpha} .
$$

A great deal of additional detail, including extension to three-state and minimal distance conical intersections (the "nearest" intersection according to some distance metric, such as RMSD) has been given previously. ${ }^{45}$ Alternate methods for mapping the intersection seam have also appeared recently. $33,36,37,52,53$

Complete active space self-consistent field (CASSCF) ${ }^{54}$ with a (4/4) active space and a $6-31 G^{*}$ basis set was employed both for intersection optimization and ab initio dynamics. Orbitals were state-averaged ${ }^{55,56}$ with equal weights. This level of treatment was benchmarked extensively for malonaldehyde in previous work, ${ }^{20}$ and shown by its consistency with multireference singles and doubles configuration interaction (MRSDCI) benchmarks to provide a faithful representation of the excited state potential energy surfaces (PESs). All electronic structure calculations were carried out with the MoLPRo package. ${ }^{57}$

Coe, Levine, Ong, and Martinez - Page 6 
Nonadiabatic molecular dynamics was performed with the ab initio multiple spawning (AIMS) technique, implemented within MolPro. ${ }^{58}$ AIMS employs an adaptive basis set of frozen Gaussians ${ }^{59}$ following classical trajectories to sample the appropriate phase space. At points of significant nonadiabatic coupling, the basis set is dynamically enlarged to allow for transfer of population between electronic states. When a new basis function is created, or "spawned", its coefficient is coupled to that of its "parent" through the time-dependent Schrödinger equation (TDSE). The AIMS wavefunction for NStates electronic states is given as:

$$
\Psi_{\text {AIMS }}(\mathbf{R}, \mathbf{r}, t)=\sum_{I}^{\text {NStates }} \Psi_{\text {AIMS }}^{\prime}(\mathbf{R}, \mathbf{r}, t)
$$

where $\mathbf{R}$ and $\mathbf{r}$ denote nuclear and electronic coordinates, respectively. The wavefunction for each electronic state is represented as a sum of $N_{I}$ complex, time-dependent Gaussians $\chi_{j}^{\prime}$ having complex, time-dependent coefficients $c_{j}^{I}$. The Gaussians themselves are parameterized by mean positions $\overline{\mathbf{R}}$, momenta $\overline{\mathbf{P}}$, fixed widths $\alpha$, and phase $\gamma$ :

$$
\Psi_{A I M S}^{I}(\mathbf{R}, \mathbf{r}, t)=\sum_{j}^{N_{t}(t)} c_{j}^{l}(t) \chi_{j}^{l}\left(\mathbf{R}, t ; \overline{\mathbf{R}}_{j}^{I}, \overline{\mathbf{P}}_{j}^{l}, \boldsymbol{\alpha}, \gamma_{j}^{l}\right) \varphi_{l}(\mathbf{r} ; \mathbf{R}) .
$$

where $\varphi_{I}$ is the wavefunction for the Ith adiabatic electronic state. The evolution of $\overline{\mathbf{R}}_{j}^{I}$ and $\overline{\mathbf{P}}_{j}^{I}$ follows the classical equations of motion corresponding to the $I$ th electronic state. The necessary forces are obtained by solving the electronic Schrodinger equation at each of the points $\overline{\mathbf{R}}_{j}^{I}$ for each time step. Thus, each of these multidimensional trajectory basis functions (TBFs) evolves on a single adiabatic electronic state, and the limit $N_{I}$ on the sum in Eq. (4) increases as new basis functions are created, i.e. "spawned," on the Ith 
electronic state. The TBFs are products of one-dimensional Cartesian Gaussian functions with time-independent widths,

$$
\chi_{j}^{\prime}=e^{i \gamma_{j}^{\prime},} \prod_{\rho=1}^{3 N} e^{-\alpha_{\rho}\left(R_{\rho}-\bar{R}_{j \rho}^{\prime}\right)^{2}+i \bar{P}_{j \rho}^{\prime}\left(R_{\rho}-\bar{R}_{j \rho}^{\prime}\right)},
$$

where $\rho$ labels the individual Cartesian coordinates of the nuclei. The coefficients $c_{j}^{\prime}$ are propagated with the TDSE, and the TBF phase evolves semiclassically (as the timeintegral of the Lagrangian). Additional detail can be found in previous review articles. ${ }^{60,61}$ For the present work, it is especially important to note that we can define the weight or "population" of any of the TBFs in a manner analogous to the Mulliken definition ${ }^{62}$ for electronic basis functions:

$$
n_{j}^{\prime}=\sum_{i}^{N_{t}(t)} \operatorname{Re}\left(\left(c_{i}^{\prime}\right)^{*} S_{i j} c_{j}^{\prime}\right)
$$

where $S_{i j}$ is the overlap matrix element between a pair of Gaussian basis functions.

Nuclear basis function widths were chosen as discussed previously, ${ }^{60}$ and the specific values used here are 30 and 6 bohr $^{-2}$ for $\mathrm{C} / \mathrm{O}$ and $\mathrm{H}$ atoms, respectively. Initial conditions for the TBF centroids were sampled randomly from the Wigner distribution ${ }^{63}$ corresponding to the ground vibronic state in the harmonic approximation. The initial basis set consisted of 128 TBFs, which after 300 fs of simulation had grown to almost 5000. The $\mathrm{S}_{0}$ minimum geometry and frequencies used to generate the Wigner distribution are obtained using MP2 with a $6-31 \mathrm{G}^{*}$ basis set.

\section{Conical Intersections in Malonaldehyde}

We first summarize some of the important geometries in the excited state dynamics of malonaldehyde, as detailed previously. ${ }^{20}$ Following excitation to $\mathrm{S}_{2}\left(\pi \pi^{*}\right)$,

Coe, Levine, Ong, and Martinez - Page 8 


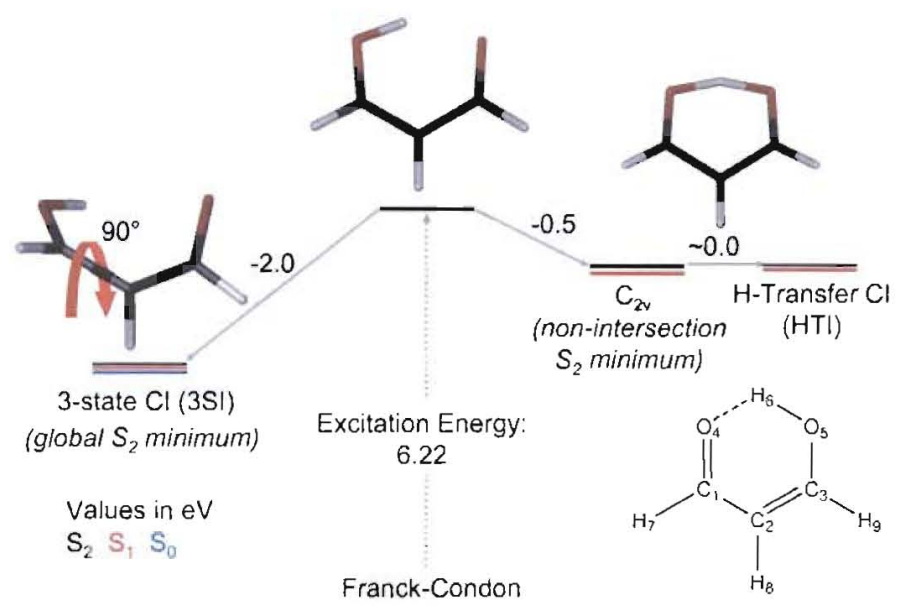

Figure 1. SA3-CAS(4/4)/6-3I $6^{\text {*t }}$ stationary points on the $S$ : potential surface. Following exeitation in the $F C$ region, there are two pathways for relaxation through conical intersection with $S_{1}$. The tirst of these involves torsion about the $C_{1}-C_{2}$ bond (see lower right for labeling convention). leading to a three-state intersection and quenching of excited state proton transfer (ESIPT). The other is closely coupled to ESIPT, and its minimal energy intersection differs from the ESIPT transition state $\left(C_{3}\right)$ only by slight displacement of $\mathrm{H}_{x}$. The $\mathrm{C}_{2}$ geometry represents the non-intersection minimum of $\mathrm{S}_{2}$.

which is the lowest optically bright excited state, there are two alternate and potentially overlapping decay pathways (see Figure 1). Each of these is characterized by a chemically distinctive $S_{2} / S_{1}$ conical intersection, located with a standard optimization procedure. ${ }^{64}$ The first of these requires little distortion from the Franck-Condon point and has $\mathrm{C}_{\mathrm{s}}$ (almost $\mathrm{C}_{2 \mathrm{v}}$ ) symmetry. Due to its geometric resemblance to the hydrogen transfer transition state on $\mathrm{S}_{0}$, we labeled it the hydrogen transfer intersection (HTI). The second intersection is much lower in energy, a twisted geometry linking the first three singlet states, i.e. an $\mathrm{S}_{2} / \mathrm{S}_{1} / \mathrm{S}_{0} \mathrm{ME}-3 \mathrm{SI} .{ }^{19}$ This ME-3SI represents the global minimum on $\mathrm{S}_{2}$ in malonaldehyde.

An additional $S_{1} / S_{0}$ MECI can be reached by simple linear interpolation starting from the ME-3SI, without breaking the $S_{1} / S_{0}$ degeneracy at any of the intervening points. Linkage of these points, demonstrated without attempt to optimize the connecting pathway, raised the prospect of extending the seam to the HTI as well, thereby connecting all of the optimized intersections among the first three singlet states. Similar 
mappings of the $S_{1} / S_{0}$ seam space in ethylene ${ }^{65}$ and fulvene ${ }^{36}$ have appeared already. Linkage of the HTI and ME-3SI would be much more surprising than the connection of the ME-3SI and $\mathrm{S}_{1} / \mathrm{S}_{0} \mathrm{MECI}$. The ME-3SI and HTI geometries are, chemically speaking, quite dissimilar. ${ }^{20}$ The $\mathrm{S}_{1} / \mathrm{S}_{0} \mathrm{MECI}$ and ME-3SI are quite similar and differ only by pyramidalization of a carbon atom. In contrast, the HTI is planar along the molecular backbone and hydrogen bonded across the mouth of the chelate ring. These differences are also reflected energetically - the ME-3SI and $\mathrm{S}_{1} / \mathrm{S}_{0} \mathrm{MECI}$ differ in energy by only $\sim 0.25 \mathrm{eV}$, whereas the difference in energies of the ME-3SI and HTI is $\sim 2 \mathrm{eV}$. As mentioned above, demonstrations of connectivity here and elsewhere ${ }^{33,36,65-69}$ feed speculation $^{21}$ that the locus of all intersection points within a molecule comprises a smooth, closed path in configuration space (possibly dissociative along some axes). According to the strongest form of this universal connectivity hypothesis, pairs of states are linked through 3 -state intersections, which then assume a pivotal role in shaping the full manifold of electronically excited states.

\section{Results and Discussion}

\section{A. Electronic Relaxation and Intersection Extrema}

AIMS population dynamics for the ensemble described above is shown in Figure 2. The lifetime of the spectroscopically bright state $\left(\mathrm{S}_{2}\right)$ is extremely brief $(\approx 60 \mathrm{fs})$, ensuring the observed broad and featureless absorption spectrum. ${ }^{70}$ An intermediate dark state also exhibits a very short lifetime of roughly $175 \mathrm{fs}$, consistent with the lack of observed fluorescence. ${ }^{71}$ At the close of 300 fs, almost $80 \%$ of the population has already 


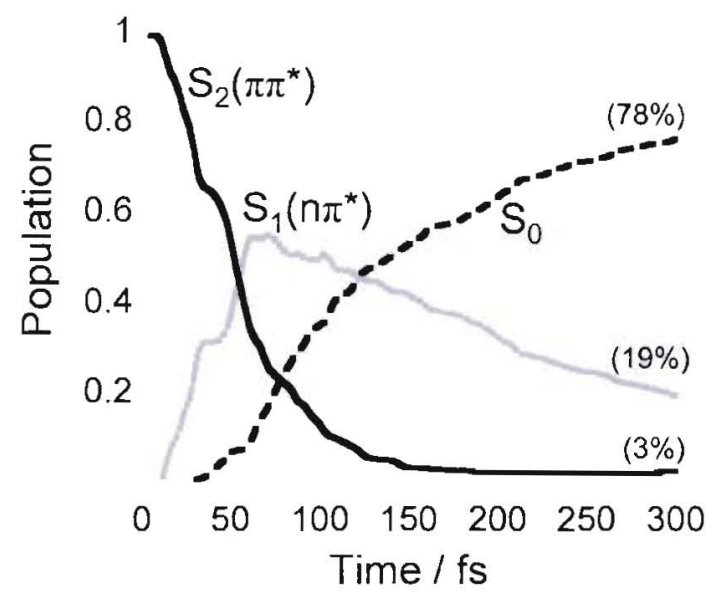

Figure 2. AIMS population dynamics for malonaldehyde, computed at the SA3CAS $(4 / 4)^{1 / 6-3} \mid G^{*}$ level. Rapid depletion of the spectroscopic state (S2) results in the experimentaliy-reported broad and featureless absomtion spectrum, while significant experimentaliy-reported broad and featureless absomtion spectrum, while significan
ground state recosery within 300 ts prevents observation of nuorescence as also has been reported experimentally. Branching ratios at the close of the simulation are given in parentheses.

made its way back to the ground state. Although the experimental data is limited in scope, that which is available is consistent with the decay rates predicted by AIMS.

As mentioned above, there are two optimized intersections that could be involved in the population loss from $\mathrm{S}_{2}$. The prospect of competition between these two decay channels is interesting given the large differences in energy, geometry, and electronic character (chemistry) of their endpoints. As we have detailed previously, ${ }^{20}$ torsion dominates and the full extent of ESIPT rises only to $\sim 20 \%$. However, on the basis of these two stationary points within the $S_{2} / S_{1}$ intersection space, one still expects to find population transferred in "clumps" surrounding torsional angles of $0^{\circ}$ and $90^{\circ}$.

Testing this hypothesis requires a means of assigning molecular properties, such as torsional angles, to population transfer events ("spawning regions," in the language of AIMS) of individual TBFs. Because population transfer takes place over an extended region of nonadiabatic coupling and a finite time span, population transfer events encompass a range of molecular geometries, energies, etc. Characterizing these extended 
regions by unique values of some property $X$ amounts to a coarse graining procedure, requiring selection of appropriate points at which to sample. We mark the beginning of an event by population loss from a basis function of $0.5 \%$ or greater over the course of a single timestep, and its end when this same quantity drops below $0.1 \%$ and the total population lost during this time is denoted as $\Delta n$. Variables within this interval are assigned unique values $X_{\Delta E_{\min }}$, coinciding with their value at the time $t_{\Delta E_{\min }}$ when the energy gap between the interacting electronic states reaches a minimum value $\Delta E_{\min }$. Typically, this coincides with the point of maximum nonadiabatic coupling over the course of the event. Generalizing to an ensemble of $N_{b f}$ basis functions, each encountering $N_{\text {events }}$ nonadiabatic events with associated population loss $\Delta n$, the amount $P_{1 \rightarrow 2}$ of population transfer binned at time $t$ and variable value $X$ becomes

$$
P_{1 \rightarrow 2}(X, t)=\sum_{i=1}^{N_{b b}} \sum_{j=1}^{N_{\text {evenns }}^{i}} \Delta n_{i j} \delta\left(t-t_{\Delta E_{\min }}^{i j}\right) \delta\left(X-X_{\Delta E_{\min }}^{i j}\right)
$$
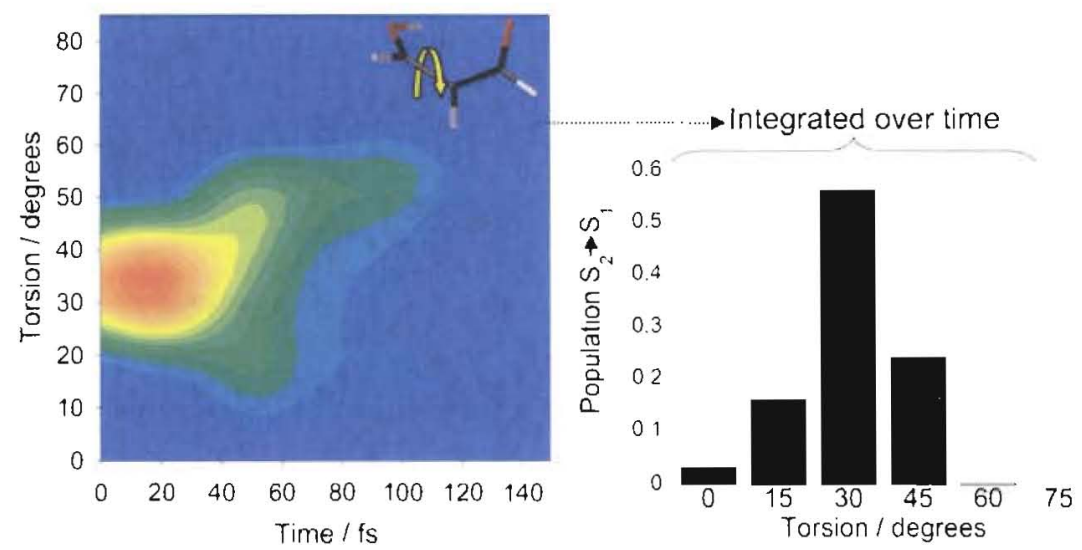

Figure 3. Lelt panel: Two-dimensional representation of $S_{2} \rightarrow S_{1}$ nonadiabatic population transfer as a function of time and torsional angle (depieted in inset). Red indicates large values of population transfer and light blue indicates zero population transfer. The procedure used for binning nonadiabatic events is deseribed in the text. Right pancl: $\mathrm{S}_{2} \rightarrow \mathrm{S}_{\text {। }}$ population transfer as a function of torsional angle only, illustrating that the bulk of electronic relaxation occurs away from both the $\mathrm{HTI}\left(0^{\circ}\right)$ and the ME-3SI $\left(90^{\circ}\right)$. 
Given this procedure for assigning a "location" to transfer of population between electronic states, we examine first the competition between $S_{2} \rightarrow S_{1}$ quenching through the HTI versus that at the $3 \mathrm{SI}$ by assigning torsional angle values to each population transfer event of the TBFs. As evidenced by the results shown in Figure 3, the prediction of a bimodal distribution, biased toward $90^{\circ}$, is not even qualitatively correct. Relatively little population is transferred near either of the $S_{2}$ intersections, and none of it occurs anywhere close (geometrically) to the true $\mathrm{S}_{2} / \mathrm{S}_{1} \mathrm{MECI}$ (the ME-3SI). Rather than clustering at $0^{\circ}$ and $90^{\circ}$, values are distributed over the $15^{\circ}-60^{\circ}$ range and concentrated between $30^{\circ}$ and $45^{\circ}$. No population transfer takes place at torsional angles greater than $60^{\circ}$, and very little $(<5 \%)$ occurs at angles less than $15^{\circ}$. In addition to highlighting the fact that torsion proceeds very rapidly (Figure 3), these facts raise the interesting question of why, if the $\mathrm{S}_{2} / \mathrm{S}_{1} \mathrm{MECIs}$ fall at $0^{\circ}$ and $90^{\circ}$, does no population transfer occur in the immediate neighborhood of those geometries?

The answer lies in remembering that conical intersections comprise $\mathrm{N}-2$ dimensional seams, not isolated points of measure zero. The intersection geometries shown in Figure 1 merely mark points on a hyperplane cutting through the full configuration space of the molecule. The point is made more forcefully by tracing a path along this hyperplane, as in Figure 4. The path was generated using the constrained optimization procedure described above, marking points along the seam in $5^{\circ}$ intervals of 


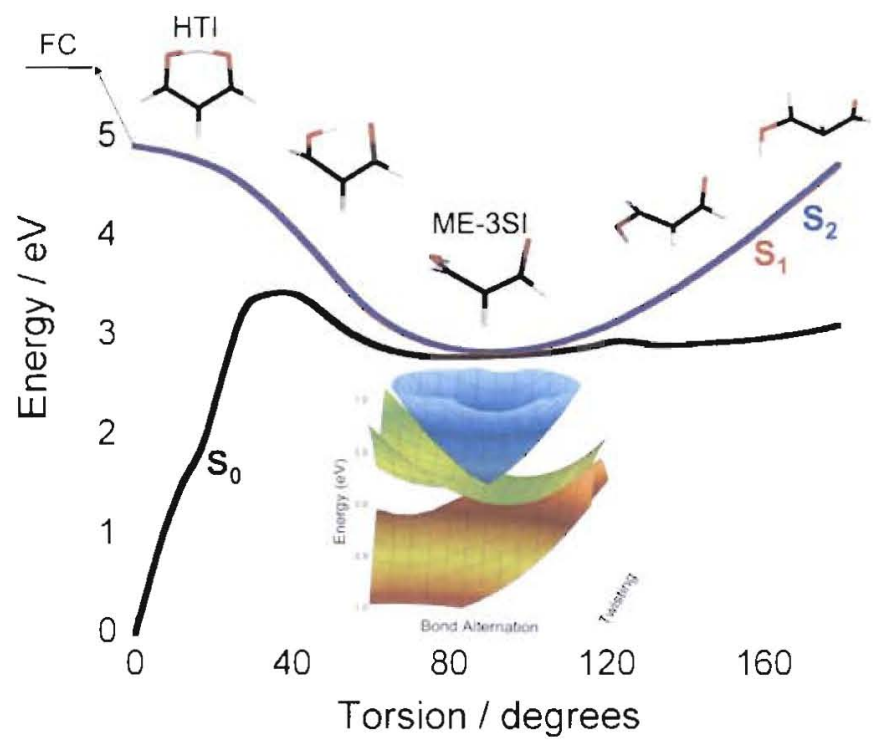

Figure 4. Connectivity of the hydrogen transfer (HITI) and inimimal energy 3-state intersection (ME-3S1) points. Constrained relaxatton in $5^{\circ}$ intersals fiom the planar to $90^{\circ}$ twisted geometries maintains the degencracy of $S_{2}$ with $S_{1}$ at all points along the connecting pathway. The Franck-Condon (FC) point is localed at $0^{\circ}$ torsion, slightly higher in energy than the HTI. Included is an approximate view of the surlace topngraphy in the vicinty of the MI:-3SI.

the $\phi_{O_{4} C_{1} C_{2} C_{3}}$ torsional angle (see Figure 1). $S_{0}$ gradually increases in energy from the planar geometry to degeneracy with $\mathrm{S}_{1}$ and $\mathrm{S}_{2}$ at the ME-3SI. Figure 4 indicates that the HTI is a saddle point in the $\mathrm{S}_{2} / \mathrm{S}_{1}$ seam space, the ME-3SI is the true minimum of the $\mathrm{S}_{2} / \mathrm{S}_{1}$ seam space, and between them lies a range of energetically accessible intersections leading away from the Franck-Condon region. Rigorous classification of the order of the saddle point in the intersection space for the HTI requires application of second-order methods for characterizing seam points in terms of mode frequencies. Such methods have recently been introduced, ${ }^{69,72-76}$ but we have yet to apply them to malonaldehyde. However, it is likely that the HTI is a first-order saddle point in the intersection space, connecting the two symmetry-equivalent ME-3SIs corresponding to clockwise and counter-clockwise torsion about the $\mathrm{C}-\mathrm{C}$ bond. The seam continues for torsional angles from $90^{\circ}$ to $180^{\circ}$. Many of these features strongly resemble those in Z-penta-3,5- 
dieniminium (ZPD), a model retinal chromophore. As with malonaldehyde, the planar intersection in ZPD is a saddle point in the $S_{1} / S_{0}$ seam space, ${ }^{33}$ and surface hops ${ }^{77}$ between states are concentrated at torsional angles of $60-80^{\circ}$ as opposed to the $\left(\sim 90^{\circ}\right.$ twisted) seam minimum. ${ }^{35}$ The seam persists for torsional angles spanning the full range $0-180^{\circ} .36$

Because discussion of intersection seams, for ease of calculation and visualization, frequently devolves into detailed consideration of their minima, it is useful to probe further the extent to which the ME-3SI typifies molecular properties at points of significant $\mathrm{S}_{2} \rightarrow \mathrm{S}_{\mathrm{I}}$ relaxation. It is clear that, geometrically speaking, population transfer occurs nowhere close to the ME-3SI, but how about energetically? How similar is the potential energy at points of significant internal conversion to that at the ME-3SI? This question is addressed in Figure 5, where the same procedure as was applied to torsion is applied to the "excess" energy, the difference in the energy of $\mathrm{S}_{2}$ at population transfer

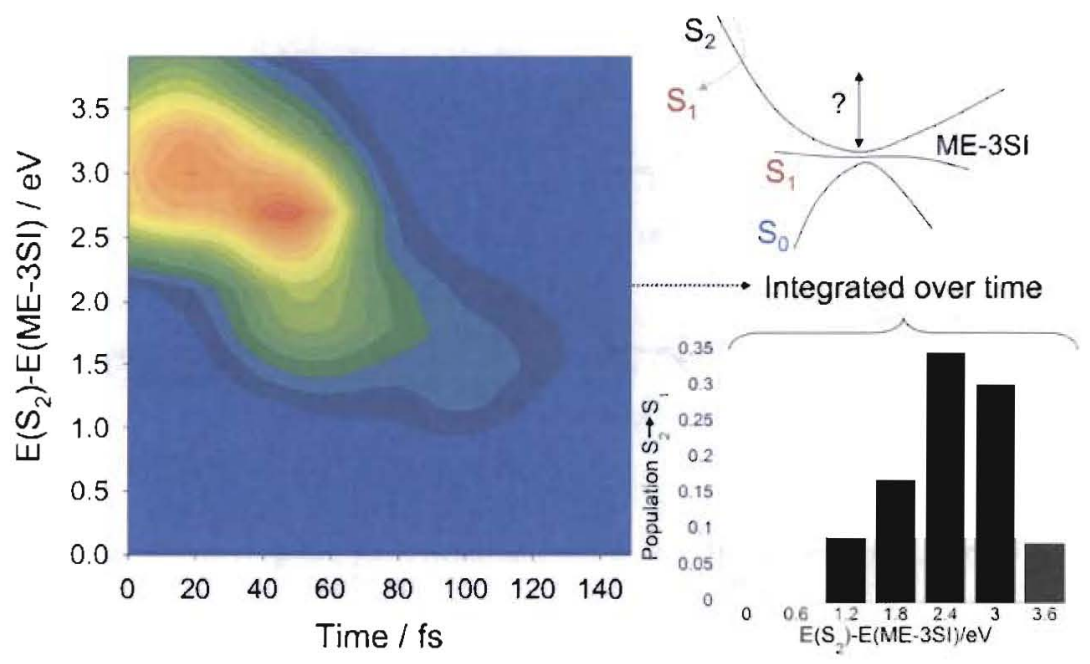

Figure 5. Left panel: Two-dimensional representation of $S_{2} \rightarrow S_{1}$ nonadiabatic population transfer as a function of time and energy in exeess of the ME-3SI. Right panel: $S_{2} \rightarrow S_{1}$ population transfer as a function of excess energy only, illustrating that all electronic relaxation occurs at energies well above that at the ME-3SI. 
points and at the ME-3SI. As is to be expected, energetic and geometric proximity to the ME-3SI are correlated, and the energetic distance decreases in time as did the geometric distance - the wavepacket is moving "downhill". Nevertheless, the wavepacket moving on $\mathrm{S}_{2}$ comes no closer than $1.2 \mathrm{eV}$ to the ME-3SI (Figure 5) prior to population being lost almost completely to $\mathrm{S}_{1}$.

Extending the view of intersection connectivity to include a vibration roughly orthogonal to torsion offers further insight into depletion of $S_{2}$ prior to the wavepacket reaching the ME-3SI. Because $\pi \rightarrow \pi^{*}$ excitation typically excites bond alternation modes, an idealized version of such a coordinate (Figure 6, left) was chosen; the resulting twodimensional view of the seam is shown in Figure 6. The orthogonal displacement corresponds roughly to a branching plane ${ }^{78}$ vector of the $\mathrm{ME}-3 \mathrm{SI},{ }^{20}$ and is closely related to a branching plane vector of the HTI (reference ${ }^{19}$, see Supporting Information) as

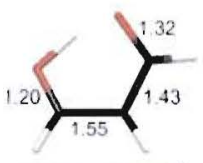

(0. $\left.-083 A^{\circ} a m u^{2}\right)$

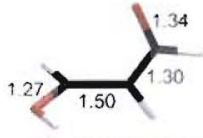

$\left(90,-0.82 A^{\cdot}\right.$ amu $\left.^{12}\right)$

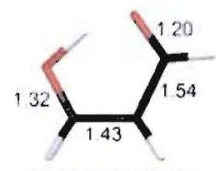

(0.083 $\mathrm{A}^{*}$ amu

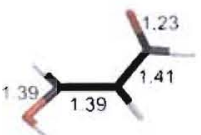

(90. $082 \mathrm{~A}^{*} \mathrm{amu}^{\prime}$ )
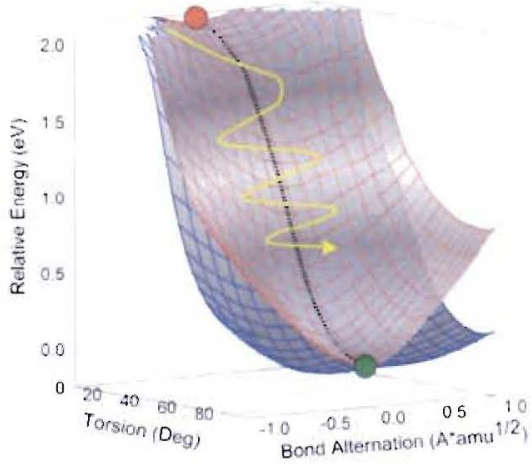

Figure 6. Two-dimensional vicw ol the $S_{2} / S_{1}$ intersection seam connecting the HTl (red circle, top) and the MF-3SI (green circle. bottom). Perlurbations along a bond alternation coordinate (illustrated at left) have been made to seam geometries along the torsional line shown in Figure 4 (depicted as a dotted line here). Because bond alternation projects onto one of the branching plane axes of both the HTI and ME-3SI, the degeneracy is broken to first order for displacements along this axis. The $S_{11}$ electronic state has been omitted for clarity, but intersects with $S_{2}$ and $S_{1}$ at the MF-3SI. The yellow line indicates a schematic of the observed dynamics which criss-crosses the seam as the molecule relaxes toward the ME-3SI.

Coe, Levine, Ong, and Martinez - Page 16 
well. Insomuch as it projects onto the branching space at any given point along the seam, it will break the degeneracy to first order in displacement. Due to the higher frequency of bond alternation modes relative to torsion $\left(\sim 300 \mathrm{~cm}^{-1}\right.$ vs. $\left.\sim 1500 \mathrm{~cm}^{-1}\right)$, the two-state intersection line is traversed multiple times as the molecule twists its way to the ME-3SI, indicated schematically by the yellow line in Figure 6. These repeated passes fully deplete $S_{2}$ population prior to $S_{0}$ becoming energetically accessible.

\section{B. Electronic Relaxation and 3-State Intersections}

Having established that the ME-3SI is not accessed on $\mathrm{S}_{2}$ prior to its depletion, it would be tempting to assume that 3 -state intersections therefore play no part in electronic relaxation. This assumption, however, falls prey to the same oversimplification as would locate the $\mathrm{S}_{2} \rightarrow \mathrm{S}_{1}$ population transfer in clusters around the HTI and ME-3SI. Just as the $\mathrm{S}_{2} / \mathrm{S}_{1}$ intersection seam cannot be reduced to two points, so the 3 -state intersection seam cannot be reduced to one point. The wavepacket's failure to reach the ME-3SI does not imply that population does not transfer near 3-state intersections located higher in energy.

In order to clarify this point, Eq. (7) was applied again to $S_{2} \rightarrow S_{1}$ nonadiabatic coupling regions, but this time for binning the energy gap separating $\mathrm{S}_{1}$ from $\mathrm{S}_{0}$. If $S_{2} \rightarrow S_{1}$ population transfer occurs when the $S_{1} / S_{0}$ gap is small, one can conclude that $S_{2}$ relaxation occurs in the vicinity of a three-state intersection. In fact, the dominant $\mathrm{S}_{1} / \mathrm{S}_{0}$ gap decreases as time progresses (left panel of Figure 7), signifying that the wavepacket is indeed approaching a region of 3-state intersections. The integrated plot (right panel of Figure 7) shows that there is a small amount of $S_{2} \rightarrow S_{1}$ transfer $(<10 \%)$ taking place at geometries with $\mathrm{S}_{1} / \mathrm{S}_{0}$ gaps of less than $0.5 \mathrm{eV}$. After this initial descent, $\mathrm{S}_{1}$ population moves away from regions where the gap with $S_{2}$ is less than $\sim 1 \mathrm{eV}$.

Coe, Levine, Ong, and Martinez - Page 17 


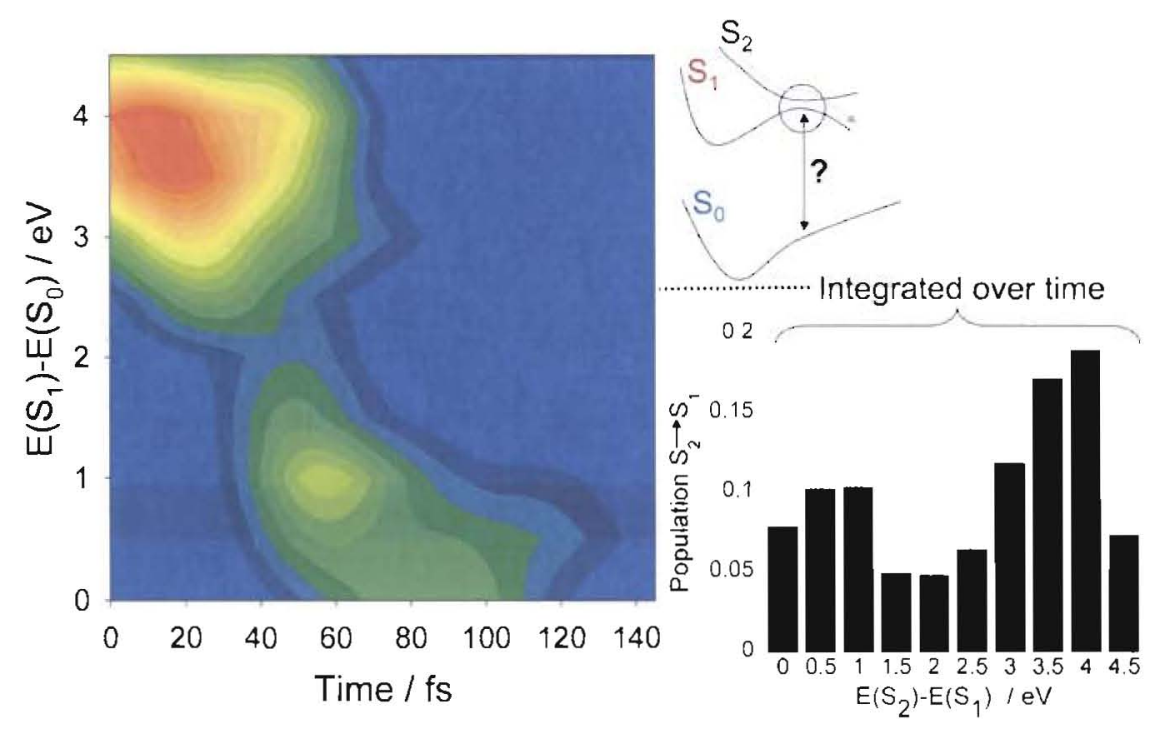

Figure 7. Left panel: Two-dimensional representation of $S_{2} \rightarrow S_{1}$ nonadiabatic population transfer as a function of time and $S_{1} / S_{11}$ energy gap. Right pancl: $S_{2} \rightarrow S_{1}$ population transfer as a function of $S_{1} / S_{0}$ energy gap only, illustrating that most $S_{2} \rightarrow S_{1}$ clectronic relaxation occurs where the $S_{1} / S_{0}$ gap is $0.5 \mathrm{cV}$ or greater.

If population reaching $S_{1}$ passes quickly on to $S_{0}$, then promotion of direct $S_{2} \rightarrow S_{0}$ decay by 3 -state intersections is definitively established. Reversing the emphasis of Figure 7, where the property of interest was $S_{1} / S_{0}$ gaps at $S_{2} \rightarrow S_{1}$ population transfer events, Figure 8 highlights $S_{2} / S_{1}$ gaps for $S_{1} \rightarrow S_{0}$ events. Roughly $1 \%$ of population is transferred to the ground state at points for which the $S_{2} / S_{1}$ gap is less than $0.5 \mathrm{eV}$ (right panel of Figure 8), but the total distribution is concentrated at energy gaps in excess of $2 \mathrm{eV}$. Inspection of the time dependence (left panel of Figure 8) shows that population transferred at small gaps does so very early (50-100 fs) in time.

A brief examination of the $S_{1}$ surface provides some insight into the absence of direct decay from $S_{2}$ to the ground state. Figure 9 details $S_{1}$ surface extrema as predicted by energy minimization. The $\mathrm{S}_{1}$ minimum, a planar and open geometry (i.e, the hydrogen bond length is considerably lengthened beyond that at the Franck-Condon point), lies well over $1 \mathrm{eV}$ below the hydrogen transfer transition state $\left(\mathrm{C}_{2}\right.$ symmetry), 

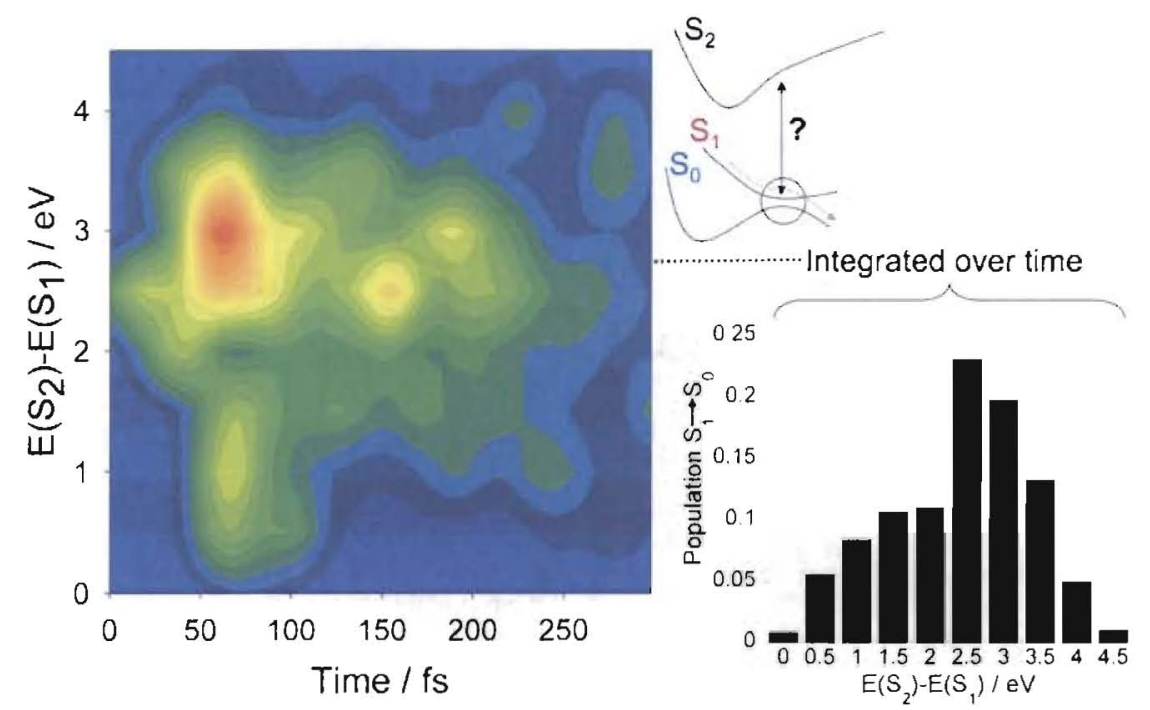

Figure 8. Left panel: Two-dimensional representation of $S_{1} \rightarrow S_{01}$ nonadiabatic population transfer as a function of time and $S_{2} / S_{1}$ energy gap. Right panel: $S_{1} \rightarrow S_{0}$ population transfer as a function of $S_{2} / S_{1}$ energy gap only, illustrating that all but a very small portion $(<1 \%)$ occurs in regions where the $S_{2} / S_{1}$ gap is $0.5 \mathrm{cV}$ or grealer.

and almost half an $\mathrm{eV}$ below the ME-3SI. The $\mathrm{C}_{2}$ geometry is, in turn, more than $1 \mathrm{eV}$ down from the HTI. Population passing from $S_{2}$ to $S_{1}$ near either of the two $S_{2} / S_{1}$ intersections (HTI or ME-3SI) will be funneled to the $\mathrm{S}_{1}$ minimum or points lying lower in the $\mathrm{S}_{1} / \mathrm{S}_{0}$ intersection space (of which the $\mathrm{S}_{1} / \mathrm{S}_{0}$ MECI may be considered representative). In either case, the population is being funneled away from regions where the $S_{2} / S_{1}$ gap is small. Thus, direct relaxation from $S_{2}$ to $S_{0}$ is unlikely. The route to sequential $\mathrm{S}_{2} \rightarrow \mathrm{S}_{1} \rightarrow \mathrm{S}_{0}$ relaxation that most looks like (and may be experimentally indistinguishable from) direct $S_{2} \rightarrow S_{0}$ decay is then $S_{2} \rightarrow S_{1}$ passage of a wavepacket traveling towards the ME-3SI followed by $\mathrm{S}_{1} \rightarrow \mathrm{S}_{0}$ passage while the wavepacket travels away from the ME-3SI. 


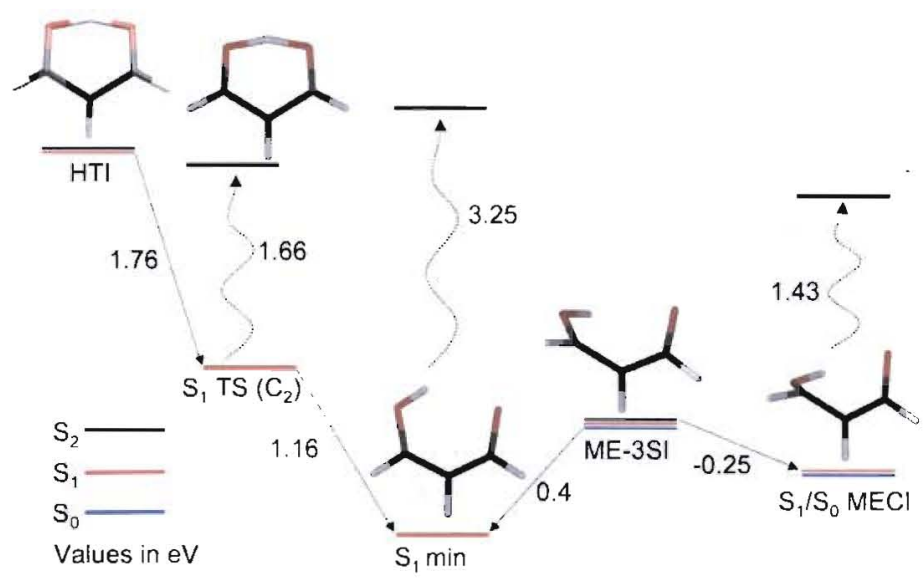

Figure 9. $S \wedge 3-C \wedge S(4 / 4) / 6-31 G^{*}$ stationary points on the $S$, polential surface. Dotted arrows denote energy gaps at a single geometry; straight arrows indicate encrgy differences for different genmetries. Energy gaps with $S_{2}$ are large at both the $S_{\text {, }}$ minimum and the $S_{1} / S_{0} M[C l$, making it likely that pieces of the wavepacket arriving on $\mathrm{S}_{1}$ from $\mathrm{S}_{2}$ will be quickly funneled away from the region near the $\mathrm{M}[3 \mathrm{Cl}$. This decreases the likelihood of direct decay to $S_{0}$ through 3 -state intersections. There is a large barrier to the $\left(\mathrm{C}_{2}\right)$ FSIPT transition stale.

Trivial extension of an argument made by Mead and Truhlar provides qualitative justification for observing minimal direct $\mathrm{S}_{2} \rightarrow \mathrm{S}_{0}$ relaxation. The original application ${ }^{79}$ was to the frequency of conical intersections relative to that of purely avoided crossings (AC), contending that appearance of an AC probably indicates that a $\mathrm{CI}$ is somewhere nearby. The total configuration space volume spanned by $d=3 N-6$ dimensions $(N \geq 3$, where $N$ is the number of atoms), each of linear extent $L$, is equal to $L^{d}$. The gap $G$ separating a conically intersecting pair of adiabatic energy levels $U_{2}$ and $U_{1}$,

$$
G \equiv U_{2}-U_{1}
$$

is less than or equal to $\varepsilon$ for a distance proportional to $\varepsilon$ along each of the two branching plane directions (the gap grows linearly along these axes, and vanishes to first order along all others), so the "volume" occupied by each intersection in this region is $V(C I)=L^{d-2} \varepsilon^{2}$. By similar reasoning, the configuration space volume per 3-state intersection is $V(3 S I)=L^{d-5} \varepsilon^{5}$. 
The total volume consumed by conical intersections (3-state intersections) is then found by multiplying $V(C D)(V(3 S I))$ by the total number of conical intersections (3-state intersections) within the full $L^{d}$ region. Assuming a density $\rho$ for zeros of electronic matrix elements gives rise to $N(C l)=\rho^{2} L^{2}$ and $N(3 S I)=\rho^{5} L^{5}$. So the total ratio of configuration space volume consumed by 3 -state intersections relative to that of conventional (2-state) intersections is

$$
\frac{V_{t o l}(3 S I)}{V_{t o l}(C I)}=\frac{N(3 S I) V(3 S I)}{N(C I) V(C I)}=\frac{\left(\rho^{5} L^{5}\right)\left(L^{d-5} \varepsilon^{5}\right)}{\left(\rho^{2} L^{2}\right)\left(L^{d-2} \varepsilon^{2}\right)}=(\rho \varepsilon)^{3} \sim \varepsilon^{3},
$$

where the final, approximate equality follows by assuming the density $\rho$ to be $O(1)$, as rationalized previously. ${ }^{79}$ This result is perfectly in keeping with the intuitive expectation that the distinction between 2- and 3-state intersections should be simply related to the difference in dimensionality (three) of their degenerate subspaces. Highlighted by this procedure, however, is the length scale dictating the precise magnitude of the difference that defining the linear region surrounding the intersection seams. While no rigorous procedure currently exists for establishing bounds on the magnitude of $\varepsilon$, we estimate its order as 0.01 (amu $)^{1 / 2}$ Angstrom on the basis of published values for previously characterized conical intersections. ${ }^{48,80}$ Comparison with the ratio of two-state intersection volume to that of purely avoided crossings,

$$
\frac{V_{t o t}(A C)}{V_{t o t}(C I)} \sim \mathcal{E}^{\frac{d}{2}-1}
$$

reveals that (except in the case of very small molecules, and hence small values of $d$ ), the ratio of three-state to two-state intersection volumes is even smaller. It would not be surprising, then, to discover that three-state intersections were everywhere present but their direct effects nowhere visible. Of course, this does not mean that the indirect effects 
of three-state intersections are unimportant - the short lifetime of $S_{1}$ (approximately 100fs from Figure 2) is due to the fact that the presence of the three-state intersection engenders large regions of closely spaced $S_{2} / S_{1}$ and $S_{1} / S_{0}$ intersections. Thus, the presence of a three-state intersection implies a higher probability than usual that population transferring from $S_{2}$ to $S_{1}$ (in the neighborhood of the three-state intersection) will come close to an $\mathrm{S}_{\mathrm{V}} / \mathrm{S}_{0}$ intersection shortly thereafter.

\section{Summary}

Visualizing conical intersection seams in many-dimensional systems is challenging in any context, and is practically impossible without drastically restricting one's view to (at most) two-dimensional subspaces. The simplest solution, adequate for many purposes, is to characterize an extended region of the seam by its local minimum at least in the sense that this geometry typifies, in some average way, those of the surrounding basin. This basin represents a locus of points at which nonadiabatic transitions concentrate. While this procedure can provide valuable guidance in building photochemical reaction pathways, it is equally conducive to the profoundly misleading impression that minimal energy points are somehow the whole story, dynamically speaking, or that they are necessarily relevant at all. Given that photochemical processes are by their very nature far from equilibrium, and most of the interesting molecules have many degrees of freedom, it can be quite difficult to make detailed predictions without extensive knowledge of the manner in which degenerate hypersurfaces (intersection spaces) criss-cross steepest descent lines and surface barriers. For processes where partial equilibration on the excited state precedes significant nonadiabatic effects, it is clear that 
the most insight will come from placement of intersection seams on free energy surfaces. ${ }^{81-83}$

Many of the distinctive features of 3-state intersections, such as their consequences for geometric phase, remain an active area of research. We have provided a detailed look at nonadiabatic dynamics not only in the presence of a 3-state intersection, but one that constitutes the minimum energy point of the lowest spectroscopically accessible excited state $\left(S_{2}\right)$. In spite of rapid ground state recovery, there is little evidence of direct $\mathrm{S}_{2} \rightarrow \mathrm{S}_{0}$ population transfer. In addition, the vast majority ( $>95 \%$ ) of population transferred between states does so at points for which the "complementary" gap (e.g., the $\mathrm{S}_{2} / \mathrm{S}_{1}$ gap in regions of $\mathrm{S}_{1} \rightarrow \mathrm{S}_{0}$ relaxation) is large $(>0.5 \mathrm{eV})$, signifying configuration space regions removed from the triply degenerate seam. Seam space dimensionality differences provide an intuitive rationale for this result, in that 3 -state degeneracies are, to first order, broken in three dimensions additional (five, total) to those breaking 2-state degeneracies. Consequently, the latter persist over a much larger fraction of configuration space. In order to reach a 3-state intersection, wavepackets must weave their way through many 2 -state intersections without losing all of their population. Even if a 3-state intersection is successfully reached from points higher in energy, the middle state must possess topography favoring rapid continuation to the next one lower in energy in order for direct transfer to occur. Nevertheless, three-state intersections are expected to have dramatic indirect effects in that their presence engenders many closely spaced twostate intersections and therefore greatly increases the likelihood of $S_{2} \rightarrow S_{1}$ transfer followed quickly by $S_{1} \rightarrow S_{0}$ transfer. This is suggested in the case of malonaldehyde by the very short $(\approx 100 \mathrm{fs})$ lifetime of the intermediate $S$ । state.

Coe, Levine, Ong, and Martinez - Page 23 
Evidence for the universality of intersection connectivity appears to be mounting. Given the machinery developed for seam following, it is possible to link chemically unrelated points in intersection spaces by pathways that may ${ }^{33,36,37}$ or may not ${ }^{45,48}$ follow steepest descent lines. Surprising examples of connectivity do not constitute proof, however, but provide motivation for a formal demonstration (or falsification) of the universal connectivity hypothesis.

\section{Acknowledgements}

This work was supported by the NSF through grants CHE-05-35640 and CHE-06-50013. JDC thanks Dr. Seth Olsen for many helpful comments improving the style and readability of the manuscript.

Coe, Levine, Ong, and Martinez - Page 24 


\section{Figure Legends}

Figure 1. SA3-CAS(4/4)/6-31G* stationary points on the $\mathrm{S}_{2}$ potential surface. Following excitation in the $\mathrm{FC}$ region, there are two pathways for relaxation through conical intersections with $S_{1}$. The first of these involves torsion about the $C_{1}-C_{2}$ bond (see lower right for labeling convention), leading to a three-state intersection and quenching of excited state proton transfer (ESIPT). The other is closely coupled to ESIPT, and its minimal energy intersection is virtually indistinguishable (geometrically and energetically) from the ESIPT transition state $\left(C_{2 v}\right)$, which also represents the nonintersection minimum of $\mathrm{S}_{2}$.

Figure 2. AIMS population dynamics for malonaldehyde, computed at the SA3CAS(4/4)/6-31G* level. Rapid depletion of the spectroscopic state $\left(\mathrm{S}_{2}\right)$ results in the experimentally-reported broad and featureless absorption spectrum, while significant ground state recovery within 300 fs prevents observation of fluorescence as also has been reported experimentally. Branching ratios at the close of the simulation are given in parentheses.

Figure 3. Left panel: Two-dimensional representation of $S_{2} \rightarrow S_{1}$ nonadiabatic population transfer as a function of time and torsional angle (depicted in inset). Red indicates large values of population transfer and light blue indicates zero population transfer. The procedure used for binning nonadiabatic events is described in the text. Right panel: $\mathrm{S}_{2} \rightarrow \mathrm{S}_{1}$ population transfer as a function of torsional angle only, illustrating that the bulk of electronic relaxation occurs away from both the HTI $\left(0^{\circ}\right)$ and the ME-3SI $\left(90^{\circ}\right)$.

Figure 4. Connectivity of the hydrogen transfer (HTI) and minimal energy 3-state intersection (ME-3SI) points. Constrained relaxation in $5^{\circ}$ intervals from the planar to $90^{\circ}$ twisted geometries maintains the degeneracy of $S_{2}$ with $S_{1}$ at all points along the connecting pathway. The Franck-Condon (FC) point is located at $0^{\circ}$ torsion, slightly higher in energy than the HTI. Included is an approximate view of the surface topography in the vicinity of the ME-3SI.

Figure 5. Left panel: Two-dimensional representation of $S_{2} \rightarrow S_{1}$ nonadiabatic population transfer as a function of time and energy in excess of the ME-3SI. Right panel: $\mathrm{S}_{2} \rightarrow \mathrm{S}_{\text {I }}$ population transfer as a function of excess energy only, illustrating that all electronic relaxation occurs at energies well above that at the ME-3SI.

Figure 6. Two-dimensional view of the $\mathrm{S}_{2} / \mathrm{S}_{1}$ intersection seam connecting the HTI (red circle, top) and the ME-3SI (green circle, bottom). Perturbations along a bond alternation coordinate (illustrated at left) have been made to seam geometries along the torsional line shown in Figure 4 (depicted as a dotted line here). Because bond alternation projects onto one of the branching plane axes of both the HTI and ME-3SI, the degeneracy is broken to first order for displacements along this axis. The $S_{0}$ electronic state has been omitted for clarity, but intersects with $\mathrm{S}_{2}$ and $\mathrm{S}_{1}$ at the ME-3SI. The yellow line indicates a schematic 
of the observed dynamics which criss-crosses the seam as the molecule relaxes toward the ME-3SI.

Figure 7. Left panel: Two-dimensional representation of $S_{2} \rightarrow S_{1}$ nonadiabatic population transfer as a function of time and $S_{1} / S_{0}$ energy gap. Right panel: $S_{2} \rightarrow S_{1}$ population transfer as a function of $S_{1} / S_{0}$ energy gap only, illustrating that most $S_{2} \rightarrow S_{1}$ electronic relaxation occurs where the $\mathrm{S}_{\mathrm{I}} / \mathrm{S}_{0}$ gap is $0.5 \mathrm{eV}$ or greater.

Figure 8. Left panel: Two-dimensional representation of $S_{1} \rightarrow S_{0}$ nonadiabatic population transfer as a function of time and $S_{2} / S_{1}$ energy gap. Right panel: $S_{1} \rightarrow S_{0}$ population transfer as a function of $S_{2} / S_{1}$ energy gap only, illustrating that all but a very small portion $(<1 \%)$ occurs in regions where the $S_{2} / S_{\text {: gap is }} 0.5 \mathrm{eV}$ or greater.

Figure 9. SA3-CAS(4/4)/6-31G* stationary points on the $S_{1}$ potential surface. Dotted arrows denote energy gaps at a single geometry; straight arrows indicate energy differences for different geometries. Energy gaps with $S_{2}$ are large at both the $S_{\text {; }}$ minimum and the $S_{1} / S_{0} M E C I$, making it likely that pieces of the wavepacket arriving on $S_{1}$ from $S_{2}$ will be quickly funneled away from the region near the $\mathrm{ME} 3 \mathrm{Cl}$. This decreases the likelihood of direct decay to $S_{0}$ through 3 -state intersections. There is a large barrier to the $\left(\mathrm{C}_{2}\right)$ ESIPT transition state. 


\section{References}

(1) Brumer, P.; Shapiro, M. Principles of the Quantum Control of Molecular Processes; Wiley-Interscience, 2003.

(2) Rice, S. A.; Zhao, M. Optical Control of Molecular Dynamics; Wiley-Interscience, 2000.

(3) Martinez, T. J. Acc. Chem. Res. 2006, 39, 119.

(4) Balzani, V.; Clemente-Leon, M.; Credi, A.; Ferrer, B.; Venturi, M.; Flood, A. H.;

Stoddart, J. F. Proc. Natl. Acad. Sci. 2006, 103, 1178.

(5) Brouwer, A. M.; Frochot, C.; Gatti, F. G.; Leigh, D. A.; Mottier, L.; Paolucci, F.;

Roffia, S.; Wurpel, G. W. H. Science 2001, 291, 2124.

(6) Kamat, P. V. J. Phys. Chem. 2007, 111C, 2834.

(7) Born, M.; Oppenheimer, J. R. Ann. Physik 1927, 84, 457.

(8) von Neumann, J.; Wigner, E. P. Phys. Z. 1929, 30, 467.

(9) Teller, E. J. Phys. Chem. 1937, 41, 109.

(10) Yarkony, D. R. Acc. Chem. Res. 1998, 31, 511.

(11) Yarkony, D. R. Rev. Mod. Phys. 1996, 68, 985.

(12) Baer, M.; Billing, G. D. The Role of Degenerate States in Chemistry; WileyInterscience, 2002; Vol. 124.

(13) Domcke, W.; Yarkony, D. R.; Koppel, H. Conical Intersections: Electronic

Structure, Dynamics, and Spectroscopy; World Scientific Publishing Company, 2004.

(14) Levine, B. G.; Martinez, T. J. Ann. Rev. Phys. Chem. 2007, 58, 613.

(15) Matsika, S. J. Phys. Chem. 2005, 109A, 7538.

Coe, Levine, Ong, and Martinez - Page 27 
(16) Matsika, S.; Yarkony, D. R. J. Chem. Phys. 2002, 117, 6907.

(17) Matsika, S.; Yarkony, D. R. J. Amer. Chem. Soc. 2003, 125, 10672.

(18) Matsika, S.; Yarkony, D. R. J. Amer. Chem. Soc. 2003, 125, 12428.

(19) Coe, J. D.; Martinez, T. J. J. Amer. Chem. Soc. 2005, 127, 4560.

(20) Coe, J. D.; Martinez, T. J. J. Phys. Chem. 2006, 110A, 618.

(21) Martinez, T. J. Far. Disc. 2004, 127, 227.

(22) Coe, J. D.; Levine, B. G.; Martinez, T. J. J. Phys. Chem. 2007, // IA, 11302.

(23) Chattoraj, M.; King, B. A.; Bublitz, G. U.; Boxer, S. G. Proc. Nall. Acad. Sci. 1996, 93,8362 .

(24) Domcke, W, Sobolewski, A. L. Science 2003, 302, 1693.

(25) Tanner, C.; Manca, C.; Leutwyler, S. Science 2003, 302, 1736.

(26) Paterson, M. J.; Robb, M. A.; Blancafort, L.; DeBellis, A. D. J. Phys. Chem. 2005, $109 \mathrm{~A}, 7527$.

(27) Lochbrunner, S.; Wurzer, A. J,; Riedle, E. J. Phys, Chem. 2003, 107A, 10580.

(28) Herek, J. L.; Pedersen, S.; Banares, L.; Zewail, A. H. J. Chem. Phys. 1992, 97, 9046.

(29) Granucci, G.; Hynes, J. T.; Millie, P.; Tran-Thi, T. H. J. Amer. Chem. Soc. 2000, $122,12243$.

(30) Aquino, A. J. A.; Lischka, H.; Hattig, C. J. Phys. Chem. 2005, 109A, 3201.

(31) Sobolewski, A. L.; Domcke, W. J. Phys. Chem. 1999, 103A, 4494.

(32) Bernardi, F; Olivucci, M.; Robb, M. A. Chem. Soc. Rev. 1996, 25, 321.

(33) Migani, A,; Robb, M. A.; Olinucci, M. J. Amer. Chem. Soc. 2003, 125, 2804.

(34) Ben-Nun, M.; Martinez, T. J. Chem. Phys, Lett. 1998, 298, 57. 
(35) Weingart, O.; Migani, A.; Olivucci, M.; Robb, M. A.; Buss, V.; Hunt, P. J. Phys. Chem. 2004, 108A, 4685 .

(36) Bearpark, M. J.; Blancafort, L.; Paterson, M. J. Mol. Phys. 2006, 104, 1033.

(37) Garavelli, M.; Page, C. S.; Celani, P.; Olivucci, M.; Schmid, W. E.; Trushin, S. A.; Fuss, W. J. Phys. Chem. 2001, 105A, 4458.

(38) Katriel, J.; Davidson, E. R. Chem. Phys. Lett. 1980, 76, 259.

(39) Han, S. S.; Yarkony, D. R. J. Chem. Phys. 2003, 119, 11561.

(40) Ichino, T.; Gianola, A. J.; Lineberger, W. C.; Stanton, J. F. J. Chem. Phys. 2006, $125,084312$.

(41) Schuurman, M. S.; Yarkony, D. R. J. Phys. Chem. 2006, 110B, 19031.

(42) Schuurman, M. S.; Yarkony, D. R. J. Chem. Phys. 2006, 124, 244103.

(43) Schuurman, M. S.; Yarkony, D. R. J. Chem. Phys. 2006, 124, 124109.

(44) Schuurman, M. S.; Yarkony, D. R. J. Chem. Phys. 2007, 127, 094104.

(45) Levine, B. G.; Coe, J. D.; Martinez, T. J. J. Phys. Chem. 2008, 112B, 405.

(46) Blancafort, L.; Robb, M. A. J. Phys. Chem. 2004, 108A, 10609.

(47) Han, S. S.; Yarkony, D. R. J. Chem. Phys. 2003, 119, 5058.

(48) Levine, B. G.; Ko, C.; Quenneville, J.; Martinez, T. J. Mol. Phys. 2006, 104, 1039.

(49) Fletcher, R. Practical Methods of Optimization, 2nd ed.; John Wiley \& Sons, 1987.

(50) Yarkony, D. R. J. Phys. Chem. 2004, 108A, 3200.

(51) Yarkony, D. R. Faraday Discuss. 2004, 127, 325.

(52) Laino, T.; Passerone, D. Chem. Phys. Lett. 2004, 389, 1.

(53) Sicilia, F.; Blancafort, L.; Bearpark, M. J.; Robb, M. A. J. Chem.Theo. Comp. 2008, 4,257 . 
(54) Roos, B. O. Adv. Chem. Phys. 1987, 69, 399.

(55) Docken, K. K.; Hinze, J. J. Chem. Phys. 1972, 57, 4928.

(56) Werner, H.-I.; Meyer, W. J. Chem. Phys. 1981, 74, 5794.

(57) MOLPRO, a package of ab initio programs designed by H.-J. Werner and P. J. Knowles, version 2002.1, R. D. Amos, A. Bernhardsson, A. Berning, P. Celani, D. L. Cooper, M. J. O. Deegan, A. J. Dobbyn, F. Eckert, C. Hampel, G. Hetzer, P. J. Knowles, T. Korona, R. Lindh, A. W. Lloyd, S. J. McNicholas, F. R. Manby, W. Meyer, M. E. Mura, A. Nicklass, P. Palmieri, R. Pitzer, G. Rauhut, M. Schütz, U. Schumann, H. Stoll, A. J. Stone, R. Tarroni, T. Thorsteinsson, and H.-J. Werner.

(58) Levine, B. G.; Coe, J. D.; Virshup, A. M.; Martinez, T. J. Chem. Phys. 2008, 347, 3.

(59) Heller, E. J. J. Chem. Phys. 1981, 75, 2923.

(60) Ben-Nun, M.; Martinez, T. J. Adv. Chem. Phys. 2002, 121, 439.

(61) Ben-Nun, M.; Quenneville, J.; Martínez, T. J. J. Phys. Chem. 2000, 104A, 5161.

(62) Szabo, A.; Ostlund, N. S. Modern Quantum Chemistry; Dover: Mineola, 1996.

(63) Hillery, M; O'Connell, R. F.; Scully, M. O.; Wigner, E. P. Phys. Rep. 1984, 106 , 121.

(64) Bearpark, M. J.; Robb, M. A.; Schlegel, H. B. Chem. Phys. Lett. 1994, 223, 269.

(65) Barbatti, M.; Paier, J.; Lischka, H. J. Chem. Phys. 2004, 121, 11614.

(66) Bearpark, M. J.; Bernardi, F.; Olivucci, M.; Robb, M. A.; Smith, B. R. J. Amer. Chem. Soc. $1995,118,5254$.

(67) Coto, P. B.; Sinicrop, A.; De Vico, L.; Ferre, N.; Olivucci, M. Mol. Phys. 2006, 104, 983.

(68) Gomez, I.; Reguero, M.; Robb, M. A. J. Phys. Chem. 2006, 110A, 3986. 
(69) Yarkony, D. R. J. Chem. Phys. 2005, 123, 204101.

(70) Seliskar, C. J.; Hoffmann, R. E. Chem. Phys. Lett. 1976, 43, 481.

(71) Arias, A. A.; Wasserman, T. A. W.; Vaccaro, P. H. J. Chem. Phys. 1997, 107, 5617.

(72) Paterson, M. J.; Bearpark, M. J.; Robb, M. A.; Blancafort, L. J. Chem. Phys. 2004, $121,11562$.

(73) Paterson, M. J.; Bearpark, M. J.; Robb, M. A.; Blancafort, L.; Worth, G. A. Phys. Chem. Chem. Phys. 2007, 7, 2100.

(74) Sicilia, F.; Blancafort, L.; Bearpark, M. J.; Robb, M. A. J. Phys. Chem. 2007, 111 A, 2182.

(75) Sicilia, F.; Bearpark, M. J.; Blancafort, L.; Robb, M. A. Theo. Chem. Acc. 2007, 118 , 241.

(76) Yarkony, D. R. J. Chem. Phys. 2005, 123, 134106.

(77) Tully, J. C. J. Chem. Phys. 1990, 93, 1061.

(78) Yarkony, D. R. J. Phys. Chem. 1997, 101A, 4263.

(79) Truhlar, D. G.; Mead, C. A. Phys. Rev. A 2003, 68, 032501.

(80) Lee, A. M. D.; Coe, J. D.; Ullrich, S.; Ho, M.-L.; Lee, S.-J.; Cheng, B.-M.; Zgierski, M. Z.; Chen, I.-C.; Martinez, T. J.; Stolow, A. J. Phys. Chem. 2007, 111 A, 11948.

(81) Burghardt, I.; Cederbaum, L. S.; Hynes, J. T. Far. Disc. 2004, 127, 395.

(82) Burghardt, I.; Hynes, J. T. J. Phys. Chem. 2006, 110 A, 11411.

(83) Burghardt, I.; Hynes, J. T.; Gindensperger, E.; Cederbaum, L. S. Phys. Scr. 2006, 73, C42.

Coe, Levine, Ong, and Martinez - Page 31 